\title{
No clear choice for imaging after first febrile UTI
}

According to a recent retrospective study published in Pediatrics, there is no ideal diagnostic algorithm for imaging infants after their first febrile UTI. La Scola et al. retrospectively assessed the ability of five guidelines-the Royal Children's Hospital of Melbourne (RCH) 2006, the National Institute of Clinical Excellence (NICE) 2007, the top down approach (TDA) 2007, the American Academy of Pediatrics (AAP) 2011, and the Italian Society of Pediatric Nephrology (ISPN) 2011-to detect vesicoureteral reflux (VUR) and scarring in febrile infants, also addressing factors such as cost and radiation burden.

A total of 304 children (aged

2-36 months) were evaluated, all of whom underwent renal tract ultrasonography and dimercaptosuccinic acid (DMSA) renal scintigraphy within the first 10 days of infection. Following a positive initial DMSA scan, patients received voiding cystourethrogram within 2 months and a second DMSA scan 12 months later (to detect scarring).

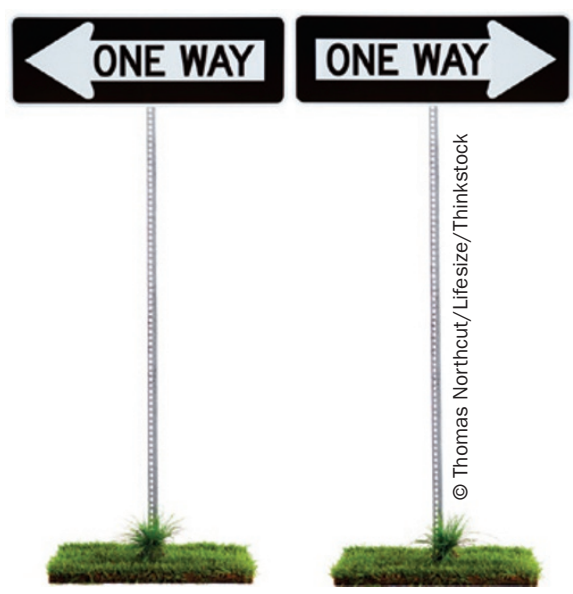

When applied retrospectively to this patient cohort, TDA 2007-which uses the initial DMSA scan results to guide further investigation-demonstrated the greatest sensitivity for detecting VUR and scarring (76\% and 100\%, respectively), but had a low specificity (50\%) and incurred substantial economic and radiation costs.
By comparison, NICE 2007 and AAP 2011 had relatively high specificities for VUR (90\%) and were shown to be the least costly in terms of financial burden (NICE 2007) and radiation exposure (AAP 2011). However, these guidelines rely on ultrasonography to select patients for further imaging, an approach that would fail to detect all cases of VUR (as ultrasonography does not reliably detect changes associated with reflux or renal damage after first febrile UTI).

With no clear choice for an optimal algorithm, the investigators recommend that sensitivity and specificity for detecting abnormalities be weighed carefully against financial cost and radiation dose on a case-by-case basis.

Melanie Clyne

Original article La Scola, C. et al. Different guidelines for imaging after first UTI in febrile infants. Pediatrics doi:10.1542/peds.2012-0164 